Article

\title{
Shannon Information Entropy in Position Space for the Ground and Singly Excited States of Helium with Finite Confinements
}

\author{
Jen-Hao Ou ${ }^{1,2, *}$ and Yew Kam Ho ${ }^{1}$ \\ 1 Institute of Atomic and Molecular Sciences, Academia Sinica, Taipei 10617, Taiwan; \\ ykho@pub.iams.sinica.edu.tw \\ 2 Department of Chemistry, National Taiwan University, Taipei 10617, Taiwan \\ * Correspondence: b02203025@ntu.edu.tw \\ Academic Editor: James F. Babb \\ Received: 13 December 2016; Accepted: 21 March 2017; Published: 24 March 2017
}

\begin{abstract}
We provide benchmark values for Shannon information entropies in position space for the ground state and ls2s ${ }^{1} \mathrm{~S}^{\mathrm{e}}$ excited state of helium confined with finite confinement potentials by employing the highly correlated Hylleraas-type wave functions. For the excited state, a "tilt" (small oscillation) on the curve of Shannon entropy as a function of width size for the confinement potential is observed. Justified by the behavior of the electron density, the localization or delocalization of the helium wave functions confined with repulsive and attractive finite oscillator $(\mathrm{FO})$ potentials are examined.
\end{abstract}

Keywords: Shannon entropy; position space; helium; Hylleraas functions; quantum confinement; finite oscillator potentials; electron density

\section{Introduction}

In recent developments on quantum information, quantum computation, quantum teleportation, and quantum cryptography, quantum entanglement plays a central role in such areas [1]. There have been considerable research activities being carried out on investigations of quantum entanglement in model, artificial, and natural atoms [2-10]. In our group, we have also carried out quantification of entanglement entropies in model [11,12] and natural atoms [13-21], such as the helium atom and the hydrogen and positronium negative ions. In these studies, the spatial (electron-electron orbital) entanglement measured with von Neumann entropy and linear entropy were quantified for the ground state, the singly excited states [13-17] and the doubly excited resonance states [18,19]. Our recent works also include quantification of entanglement entropies for atoms embedded in Debye plasmas in which the Coulomb potentials for the two-body interactions are replaced by screened Coulomb (Yukawa-type) potentials [20]. In addition to von Neumann entropy, we also reported an investigation of Shannon information entropies for two-electron atomic systems [19,21]. Shannon entropy for hydrogen-like and helium-like atomic systems with infinite confinement were investigated by Sen [22]. Recently, the von Neumann entropy for helium with finite confinement [23] and with infinite confinement were calculated by Koscik and Saha [24].

In other related development, spectroscopic atomic structures for confined atomic systems have been under active investigations [25-31]. In Ref. [23,24], the von Neumann and linear entropies of the ground state helium inside a spherical cavity with finite or infinite confinements were calculated, but Shannon information entropy for helium with finite confinement has not been reported in the literature, to the best of our knowledge. This work presents such an investigation. Here, we report calculations of Shannon entropy for the ground state and first singlet-spin excited state of helium confined with finite attractive [27-29] and repulsive potentials [24] by employing highly correlated and 
extensive Hylleraas-type wave functions. We use the finite oscillator $(\mathrm{FO})$ potentials for the external confinement potentials [24,27-29].

Shannon entropy was first introduced by Claude E. Shannon [32] in 1948 to quantify the average amount of information in a source and to evaluate probability distribution of a sample, and it also shows statistical correlations between particles [33,34]. Investigations on Shannon information entropy of atomic systems have also been reported in the literature [35]. For instance, Gadre et al. investigated Shannon entropy within Thomas-Fermi theory and for harmonic oscillator and a series of atoms [36,37]. Besides, Yanez et al. calculated Shannon entropies for the D-dimensional harmonic oscillator and hydrogen atom [38]. In our present work for the confined helium atom in its ground and excited states, Shannon entropy provides information about the localization or delocalization of this two-electron system.

\section{Theoretical Method}

The non-relativistic Hamiltonian (in atomic units) describing the helium atom is

$$
H=-\frac{1}{2} \nabla_{1}^{2}-\frac{1}{2} \nabla_{2}^{2}-\frac{2}{r_{1}}-\frac{2}{r_{2}}+\frac{1}{r_{12}}+V_{\text {external }}\left(r_{1}\right)+V_{\text {external }}\left(r_{2}\right)
$$

where the subscript 1 and 2 denote the electron 1 and 2, respectively, and $r_{12}$ is the relative distance between the electron 1 and 2. Energies in atomic units are used in the present work. The external confinement potential can be attractive, $V_{\text {att }}(r)$, or repulsive, $V_{\text {rep }}(r)$. The attractive confinement potential is described as

$$
V_{\text {att }}(r)=-A\left(1+\frac{B}{\sqrt{A}} r\right) e^{-(B / \sqrt{A}) r}
$$

and was used in previous studies for structural variations for bound $[27,29]$ and resonance states $[28,29]$. For the case with external repulsive potential,

$$
V_{\text {rep }}(r)=A-A\left(1+\frac{B}{\sqrt{A}} r\right) e^{-(B / \sqrt{A}) r}
$$

it was used in Ref. [24] for von Neumann entropy calculations on confined ground state helium. The potentials in the form of Equations (2) and (3) are called the finite oscillator (FO) potential [27]. In both cases, the parameter $A$ and $1 / B$ controls the depth and width of the potential, respectively. The tilizations of these two potentials are scattered independently in different publications [24,27-29] but no comparison was made. Therefore, we unify them together in this context to provide a thorough investigation of the difference between them. It turns out that given the same set of confinement parameters $(A$ and $1 / B)$, the energy of a specific state subjected to repulsive potential is larger than that subjected to attractive potential by $2 A$. On the other hand, entropies for both cases are the same. This may indicate that these two potentials provide similar confinement effects if parameter sets are identical.

In the present work, for $S$-states in the helium atom, we employ Hylleraas-type wave functions to describe the coordinate part of the system to take into account the correlation effects, and they are expressed as

$$
\Psi_{k m n}=\sum_{k m n} C_{k m n}\left(\exp \left[-\alpha\left(r_{1}+r_{2}\right)\right] r_{12}^{k} r_{1}^{m} r_{2}^{n}+(1 \leftrightarrow 2)\right)
$$

with $k+m+n \leq \omega$, and $\omega, k, m$, and $n$ are positive integers or zero. For the singlet-spin ground state, we have $k \geq m$. In Equation (4), $\alpha$ is a variational parameter. For a multi-electron system, the Shannon information entropy in position space, denoted as $S_{r}$, is defined by the one-electron charge density $\rho(r)$, after averaging out the rest of other electrons, as

$$
S_{r}=-\int_{0}^{\infty} \rho(r) \ln \rho(r) 4 \pi r^{2} d r
$$


where, in our present case for a two-electron system, the function $\rho(r)$ is shown as

$$
\rho\left(r_{1}\right)=\frac{2 \pi}{r_{1}} \int_{0}^{\infty} \int_{\left|r_{1}-r_{2}\right|}^{r_{1}+r_{2}}|\Psi|^{2} r_{2} d r_{2} r_{12} d r_{12}
$$

The integral in Equation (5) is solved by the Gauss-Laguerre quadrature integration. The convergence of the numerical calculation is tested by the normalization condition:

$$
\int_{0}^{\infty} \rho(r) 4 \pi r^{2} d r=1
$$

In this work, we calculate Shannon entropy in position space $\left(S_{r}\right)$ for the ground state and the $1 \mathrm{~s} 2 \mathrm{~s}{ }^{1} \mathrm{~S}^{\mathrm{e}}$ excited state of the quantum confined helium atom.

\section{Calculations and Results}

\subsection{Shannon Entropy for the Ground State and the $1 \mathrm{~s} 2 \mathrm{~s}^{1} S^{e}$ Excited State of He without Confinement}

In order to check the accuracy of our present results, first we calculate $S_{r}$ for the free-helium case. The wave functions of the ground state helium are constructed by using highly correlated Hylleraas-type function with optimal $\alpha=2$. As the value of $\omega$ in Equation (4) increases, the size of the basis set gets larger and the wave functions would be able to provide a better description for the ground state. Furthermore, by systematically changing the value of $\omega$, we have examined the optimized energy for that state, and with which the Shannon entropy in position space for such state is calculated. In Table 1, we show the convergence of the ground state energy in terms of $\omega$. It is seen that our energies compare quite well with other accurate calculations in the literature [21,39].

Once the wave functions are obtained, we apply them to calculate Shannon entropy in position space, and the results are also shown in Table 1. It is seen that both energy and Shannon entropy show good convergence. In Figure $1 \mathrm{a}, \mathrm{b}$ respectively, we show the convergence behaviors for energy and $S_{r}$. We conclude that our $S_{r}$ for the free helium ground state is 2.7051028 , and we estimate the uncertainty is within a few parts in the last quoted digit. Our present results are consistent with the earlier investigation [21].

Table 1. The ground state and $1 \mathrm{~s} 2 \mathrm{~s}^{1} \mathrm{~S}^{\mathrm{e}}$ excited-state energy and Shannon entropy of free helium.

\begin{tabular}{cccccc}
\hline \multirow{2}{*}{$\omega$} & \multirow{2}{*}{$\boldsymbol{N}$} & \multicolumn{2}{c}{ Ground State } & \multicolumn{2}{c}{ 1s2s $\mathbf{1}^{\mathbf{e}}$ Excited State } \\
\cline { 3 - 6 } & & Energy & Shannon Entropy & Energy & Shannon Entropy \\
\hline 9 & 125 & -2.903724233 & 2.70510325 & -2.145945902 & 5.4877391 \\
10 & 161 & -2.903724300 & 2.70510306 & -2.145968846 & 5.4908525 \\
11 & 203 & -2.903724334 & 2.70510296 & -2.145972626 & 5.4916522 \\
12 & 252 & -2.903724352 & 2.70510291 & -2.145973707 & 5.49189461 \\
13 & 308 & -2.903724362 & 2.70510288 & -2.145973920 & 5.49195037 \\
14 & 372 & -2.903724367 & 2.70510286 & -2.145973988 & 5.49196539 \\
15 & 444 & -2.903724371 & 2.70510285 & -2.145974012 & 5.49196837 \\
\multicolumn{2}{r}{ Ref. [21] } & -2.9037243768 & 2.7051028 & & \\
\multicolumn{2}{l}{ Ref. [39] } & -2.9037243770341195 & & -2.145974046054419 & \\
\hline
\end{tabular}

As for the $1 \mathrm{~s} 2 \mathrm{~s}{ }^{1} \mathrm{~S}^{\mathrm{e}}$ excited state of free $\mathrm{He}$, we first conduct a variational optimization of the parameter $\alpha$ to obtain better wave functions describing the $1 \mathrm{~s} 2 \mathrm{~s}^{1} \mathrm{~S}^{\mathrm{e}}$ excited state. The optimal $\alpha$ is found to be 1.19. Then, the convergence of energy and $S_{r}$ with respect to the enlargement of $\omega$ is also investigated and displayed in Table 1 . We estimate that our $S_{r}$ for the $1 \mathrm{~s} 2 \mathrm{~s}{ }^{1} \mathrm{~S}^{\mathrm{e}}$ excited state of helium is 5.49197, with an uncertainty of a few parts in the last quoted digit. 

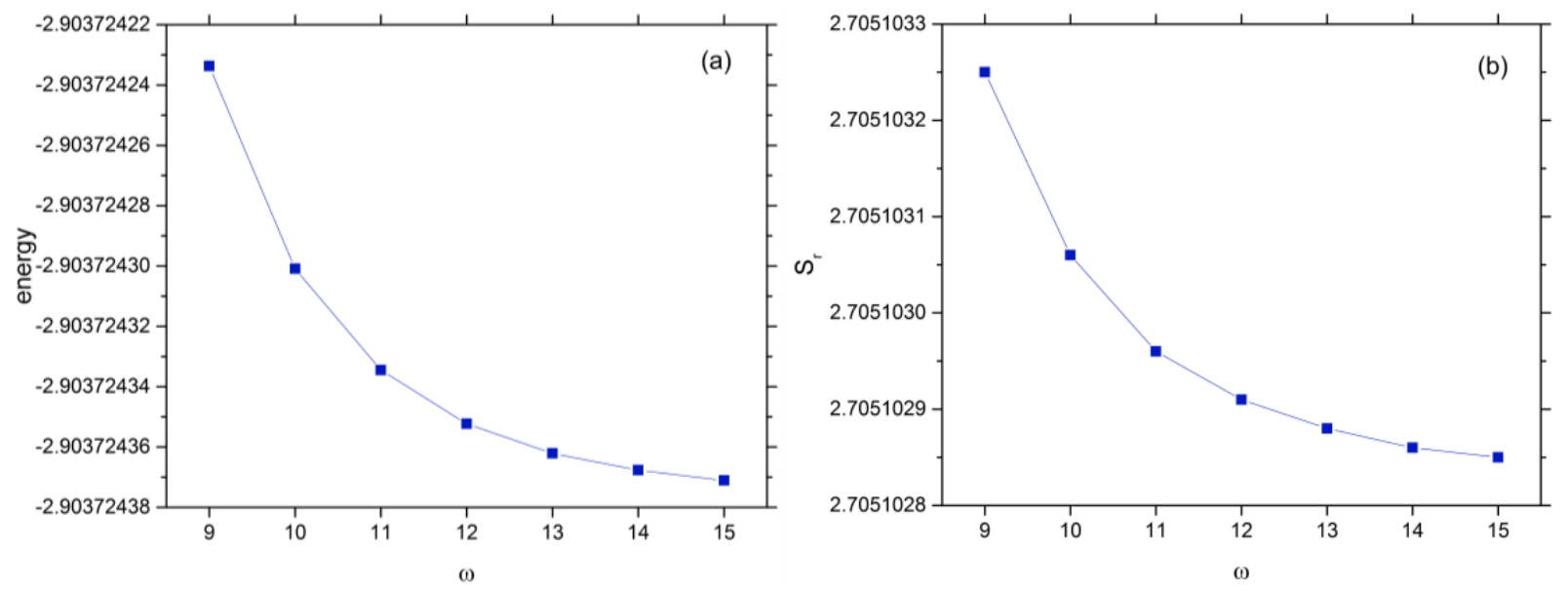

Figure 1. Energy (a) and Shannon entropy (b) for the ground state helium as functions of size of basis set governed by $\omega$.

\subsection{Shannon Entropy for Ground State He under Confinement with Attractive Potential}

Next, we consider the ground state helium confined by an external attractive potential with finite height (Figure 2), called "finite oscillator (FO) potential" [27,28] of the form given in Equation (2). Hylleraas-type wave functions as those for the free helium case, with up to $\omega=11$ ( $N=203$ terms) are used, for practical purposes to strike a balance between saving computer time and achieving acceptable accuracy. The energy for the quantum confined helium with attractive confinement for selected $A$ and $1 / B$ values are shown in Table 2 and Figure 3. Comparisons are made for selected energy values with other calculations [29], and it is seen that our energies are well in agreement with other earlier results in the literature. In the two limiting cases when the cavity size is very small or very large, the helium atom behaves like a free atom. At the small cavity limit, the energy of the system is approaching the free helium energy. At the large cavity limit, the total energy of the system is the sum of the free helium energy plus the confinement potential energy for two electrons, $-2 A$. Our calculated results support such a physical phenomenon.

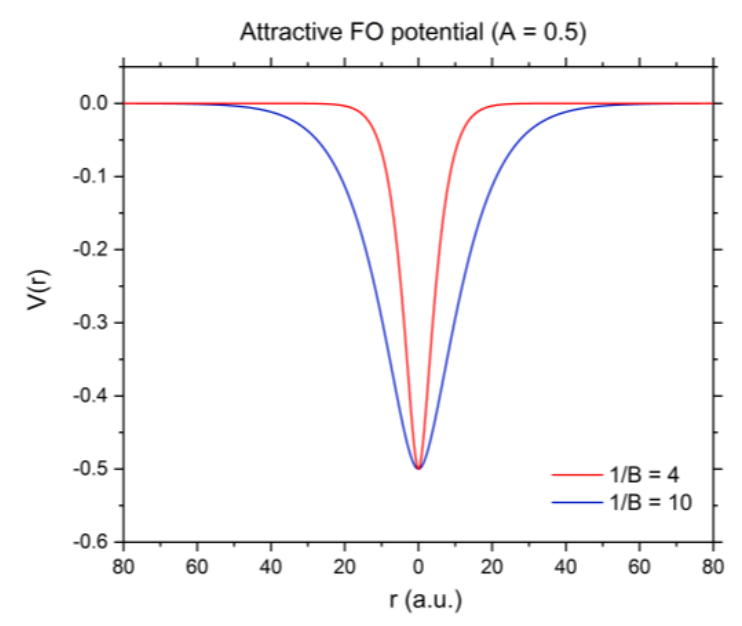

Figure 2. The finite oscillating potentials with attractive confinement for different cavity sizes $(1 / B)$, with $A=0.5$. 
Table 2. The ground state energy and Shannon entropy of helium confined with attractive potentials, with $\omega=11$.

\begin{tabular}{ccccc}
\hline \multirow{2}{*}{$\mathbf{1} \boldsymbol{B}$} & \multicolumn{2}{c}{ Energy } & \multicolumn{2}{c}{ Shannon Entropy } \\
\cline { 2 - 5 } & $\boldsymbol{A}=\mathbf{0 . 2}$ & $\boldsymbol{A}=\mathbf{0 . 5}$ & $\boldsymbol{A}=\mathbf{0 . 2}$ & $\boldsymbol{A}=\mathbf{0 . 5}$ \\
\hline 0.01 & -2.903730428 & -2.903782320 & 2.7050970 & 2.7050463 \\
\hline 0.04 & -2.904046799 & -2.906501450 & 2.7047890 & 2.7024115 \\
\hline 0.1 & -2.907290165 & $\begin{array}{c}-2.930040819 \\
-2.93004^{\dagger}\end{array}$ & 2.7016791 & 2.6804318 \\
\hline 0.25 & -2.930892544 & -3.059472114 & 2.6806153 & 2.5789191 \\
\hline 0.4 & -2.965954073 & -3.206030252 & 2.6536356 & 2.5005110 \\
\hline 1 & -3.093344574 & $\begin{array}{c}-3.564904622 \\
-3.56490^{+}\end{array}$ & 2.5989837 & 2.4661404 \\
\hline 2.5 & -3.222312613 & -3.7981899998 & 2.6227726 & 2.5795868 \\
\hline 4 & -3.261404222 & -3.853009590 & 2.6514702 & 2.6329358 \\
\hline 10 & -3.294341118 & $\begin{array}{c}-3.893533087 \\
-3.89353^{+}\end{array}$ & 2.6895775 & 2.6872398 \\
\hline 5 & -3.301992742 & -3.901931164 & 2.7018817 & 2.7016811 \\
\hline 40 & -3.303022766 & -3.903007067 & 2.7037593 & 2.7037067 \\
\hline 100 & -3.303607882 & -3.903606833 & 2.7048735 & 2.7048699 \\
\hline
\end{tabular}

Notes: ${ }^{\dagger}$ Ref. [29] (Hylleraas functions).

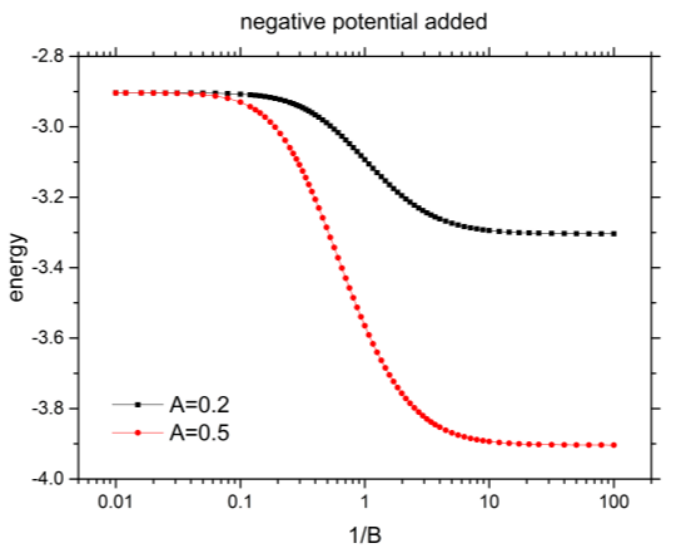

Figure 3. The ground state energy of helium with attractive confinement as a function of the cavity size $1 / B$.

Calculated for the first time, our results for Shannon entropy for ground state of He under confinement with attractive potential is shown in Table 2 and Figure 4 . Two different cases, with $A=0.2$ and 0.5 , and $1 / B$ spreading around from very small cavity size $(1 / B=0.01)$ to very large cavity $(1 / B=100)$, are reported. Shannon entropy shows a decreasing trend as the size of the cavity starts increasing from the free helium case. When the cavity is increased to a certain value, $S_{r}$ reaches a minimum. If the cavity size is increased further, $S_{r}$ turns to show an increasing trend, and eventually it is increased back to the value for the free helium case. Such a phenomenon can be qualitatively explained as follows: when the size of the cavity starts to increase, the edge of the potential barrier will have interference effect with the wave function of the ground state helium. As a result, the system would exhibit a more localized (decreasing $S_{r}$ ) character. When the cavity size is increased further after $S_{\mathrm{r}}$ reaching a minimum, it turns to show an increasing trend, indicating that the interference effect starts to diminish, and the system then becomes more delocalized. Eventually, $S_{r}$ would increase back to the value for a free helium when the edge of the cavity is far away from the effective region for the ground state helium wave function. 


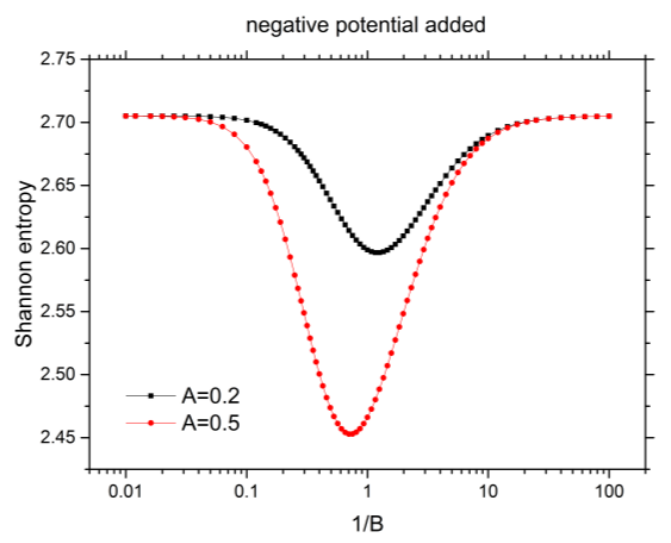

Figure 4. Shannon entropy in position space for the ground state helium with attractive confinement, shown as a function of $1 / B$.

The reasoning above is supported by the behavior of electron density, $\rho(r)$ (Equation (6)) as a function of the radius, $r$. As shown in Figure 5 , either the cavity width is very small $(1 / B=0.01)$ or very large $(1 / B=100)$, the electron density is more spread out, or more delocalized as if the helium is free. When the cavity size $(1 / B=0.702)$ matches the wave function, the electron density is the most localized toward the origin.

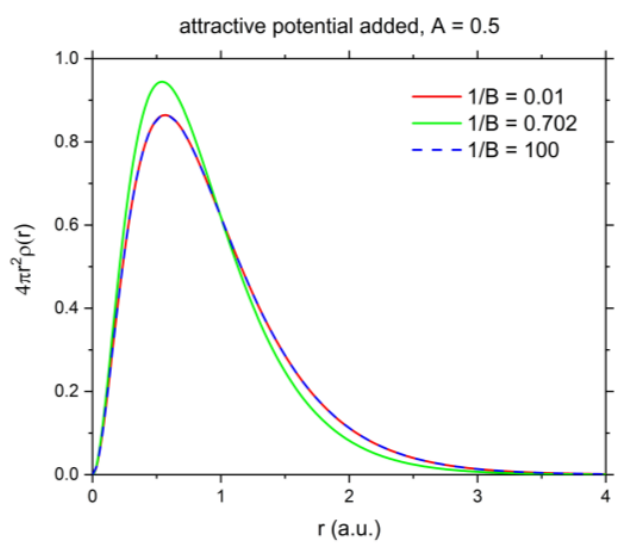

Figure 5. The electron density subjected to different cavity width $(1 / B)$ as a function of radius. The one for $1 / B=100$ (large width limit) is represented in blue dash since it is very similar to the red one for $1 / B=0.01$ (small width limit). The green curve $(1 / B=0.702)$ shows the most localized behavior, corresponding to the valley of the red curve in Figure 4.

\subsection{Shannon Entropy for the $1 s 2 s{ }^{1} S^{e}$ Excited State of He under Confinement with Attractive Potential}

In order to provide a better description of the $1 \mathrm{~s} 2 \mathrm{~s}{ }^{1} \mathrm{~S}^{\mathrm{e}}$ excited state of $\mathrm{He}$, the size of the Hylleraas-type wave function is enlarged up to $\omega=13(N=308$ terms). The energy curve is shown in Figure 6. At the large cavity limit, the energy is lowered by $2 A$ as in the ground state. Compared with the wave function for the ground state, the one for $1 \mathrm{~s} 2 \mathrm{~s}{ }^{1} \mathrm{~S}^{\mathrm{e}}$ excited state has one more node. Here comes the difference. It exhibits additional interference with the cavity. This phenomenon is probed by the "tilt" (small oscillation) or the not-so-smooth change in the slope of Shannon entropy curve from $1 / B=0.1$ to 0.72 in Figure 7 , for the $A=0.5$ case. The electron density also provides a better understanding to such phenomenon. As shown in Figure 8, the electron density is the most delocalized when the cavity width is small $(1 / B=0.015)$. As the width begins to increase from $1 / B=0.268$ to 1.14 , the electron density flows toward the origin and becomes most localized when $1 / B=2.812$. The selected values of energy and Shannon entropy are listed in Table 3. 


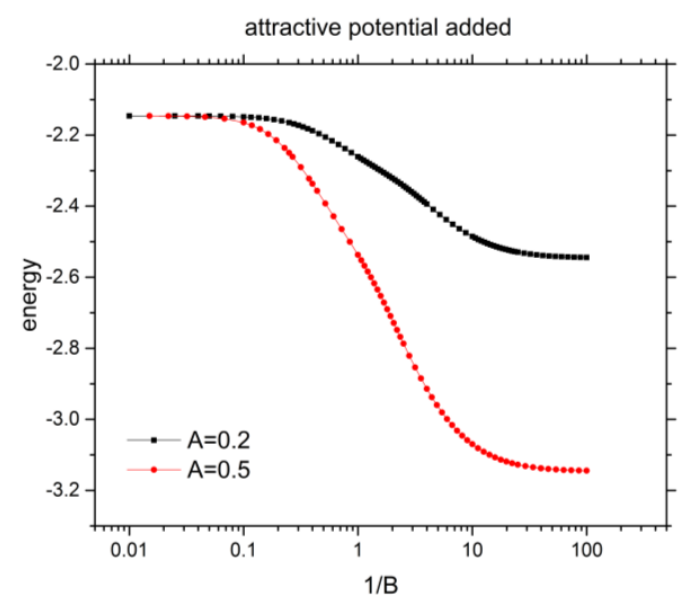

Figure 6. The 1s2s state energy of helium with attractive confinement as a function of the cavity size $1 / B$.

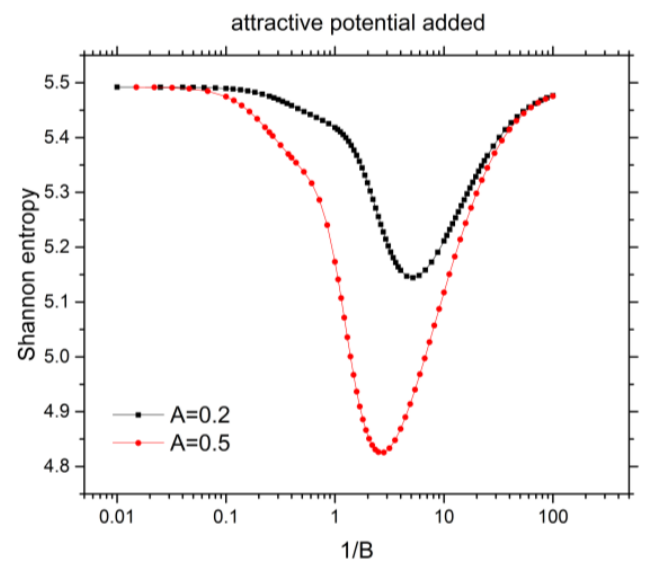

Figure 7. Shannon entropy for the 1s2s excited-state helium with attractive confinement, shown as a function of $1 / B$. The "tilt" from $1 / B=0.1$ to 0.72 (for $A=0.5$ case) resulted from the interference due to the confined cavity is apparent.

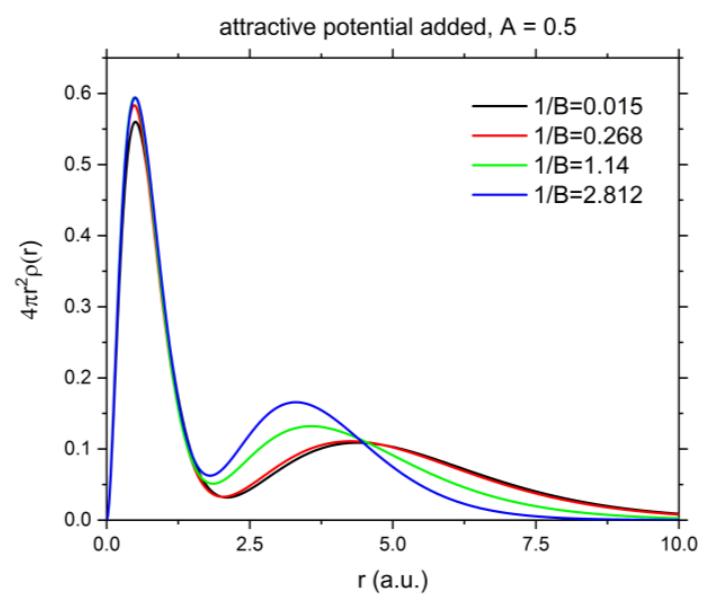

Figure 8. Electron density of specific $A$ and $1 / B$ for the $1 \mathrm{~s} 2 \mathrm{~s}^{1} \mathrm{~S}^{\mathrm{e}}$ excited-state helium with attractive confinement as a function of radius. The black curve is the small cavity limit, where the electrons are more delocalized. The blue curve $(1 / B=2.812)$ corresponds to the valley point $(A=0.5)$ in Figure 7 and is the most localized and concentrated. 
Table 3. The 1s2s state energy and Shannon entropy of helium confined with attractive potentials, with $\omega=13$.

\begin{tabular}{ccccc}
\hline \multirow{2}{*}{$\mathbf{1} \boldsymbol{B}$} & \multicolumn{2}{c}{ Energy } & \multicolumn{2}{c}{ Shannon Entropy } \\
\cline { 2 - 5 } & $\boldsymbol{A}=\mathbf{0 . 2}$ & $\boldsymbol{A}=\mathbf{0 . 5}$ & $\boldsymbol{A}=\mathbf{0 . 2}$ & $\boldsymbol{A}=\mathbf{0 . 5}$ \\
\hline 0.01 & -2.145978326 & & 5.4919460 & \\
0.015 & & -2.146108395 & & 5.4918183 \\
0.1 & -2.148528796 & -2.164613565 & 5.4895009 & 5.4745971 \\
0.25 & -2.164822224 & -2.249875684 & 5.4752129 & 5.4097138 \\
0.4 & -2.187604871 & -2.337051173 & 5.4583644 & 5.3631936 \\
1 & -2.260693038 & -2.537147708 & 5.4182621 & 5.1733749 \\
2.5 & -2.342674793 & -2.786896138 & 5.2556723 & 4.8262175 \\
4 & -2.394029709 & -2.914053557 & 5.1576614 & 4.8685244 \\
10 & -2.485237303 & -3.069904076 & 5.2113530 & 5.1173708 \\
25 & -2.529366979 & -3.127321718 & 5.3667965 & 5.3446117 \\
40 & & -3.137714313 & & 5.4151191 \\
41.38 & -2.538783608 & & 5.4270311 & \\
100 & -2.544528698 & -3.144476898 & 5.4764372 & 5.4755194 \\
\hline
\end{tabular}

\subsection{Shannon Entropy for Ground State of He under Confinement with Repulsive Potential}

Next, we consider the ground state helium confined by an external repulsive potential with finite height (see Figure 9). We use a modified finite oscillator (FO) potential of the form given in Equation (3), and this form of repulsive potential was also used in Ref. [24]. It is worth noting that the energy of a specific state with current repulsive potential is larger than that with previous attractive potential by $2 A$, while entropies for both cases with identical parameters would be the same. Again, we use similar Hylleraas-type wave functions as those for the free-helium case, with up to $\omega=11$ $(N=203$ terms). Table 4 shows the energy and Shannon entropy for quantum confined ground state helium with repulsive confinement for selected $A$ and $1 / B$ values. We choose $A=2.0$ and 5.0, the same as those used in Ref. [24]. Comparisons are made for energy with other calculations [24] and shows good agreement. When the cavity size is very small, the confinement energy is $+2 A$, and the total energy of the system is the sum of the free helium energy plus this additional amount of $2 A$, as shown in Figure 10. At the limiting case when the cavity size is very large, the repulsive potential becomes negligible, and the total energy is approaching the energy of a free helium atom. Our numerical calculated results for energies do support such conclusion.

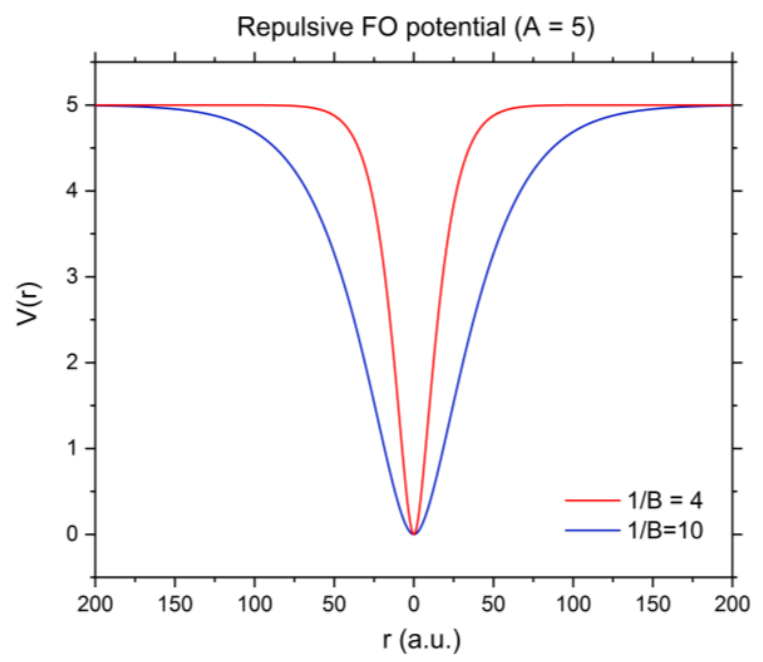

Figure 9. Finite oscillating potential with repulsive confinement, with $A=5$. 
Table 4. The ground state energy and Shannon entropy of helium confined with repulsive potentials, with $\omega=11$.

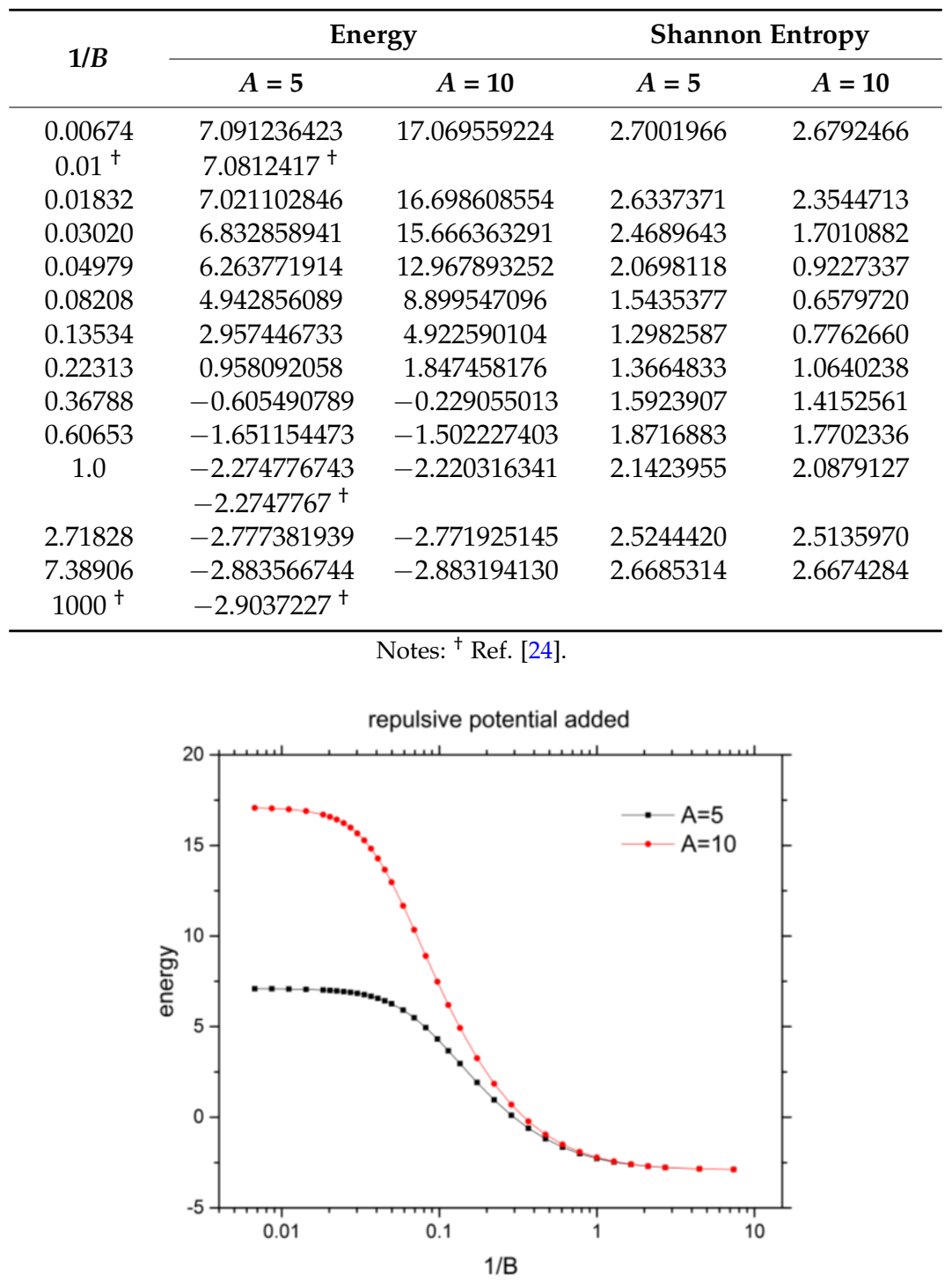

Figure 10. The ground state energy of helium with repulsive finite confinement as a function of the cavity size $1 / B$.

As for Shannon entropy for the case with an external repulsive potential, shown in Figure 11 and Table 4, the variation of $S_{r}$ for changing cavity size is very similar to that for the case with attractive potential. It first shows a decreasing trend when the cavity size starts to increase, a consequence of interference effect from the edge of the potential well on the helium wave function, leading to more localized character for the confined helium. After $S_{r}$ reaching a minimum as $1 / B$ is continued to increase, it turns to show an increasing trend, and eventually $S_{r}$ would increase back to the value for a free helium when the cavity size becomes very large. The same phenomenon can be explained in detail similar to that for the case with an external attractive potential. By observing the electron density in Figure 12 , when the width is very small $(1 / B=0.01)$ or very large $(1 / B=10)$, the electron density is similar and behaves like that of free He. However, when the width $(1 / B=0.082)$ is appropriate to the wave function, the electron density is the most localized and shows a concentrated peak. 


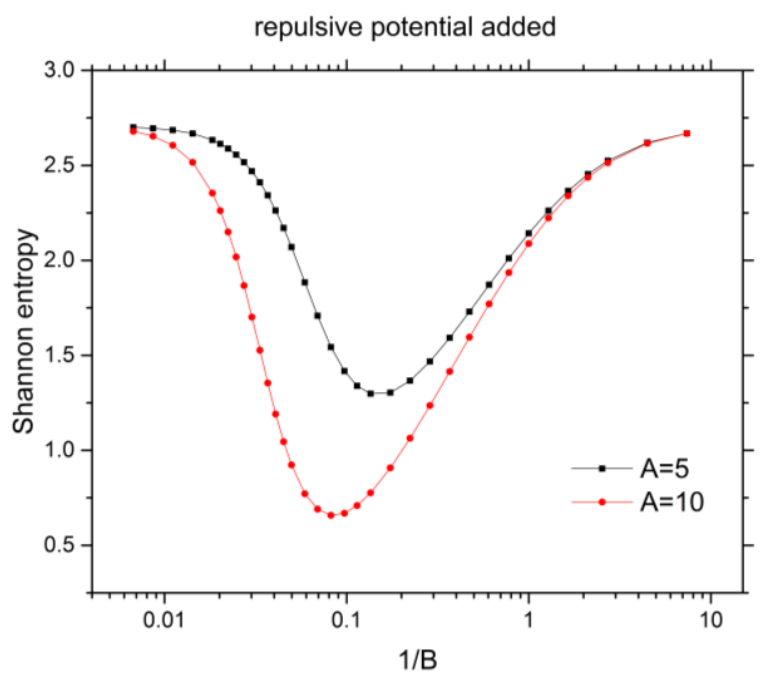

Figure 11. Shannon entropy for the ground state helium with repulsive confinement, shown as a function of the cavity size, $1 / B$.

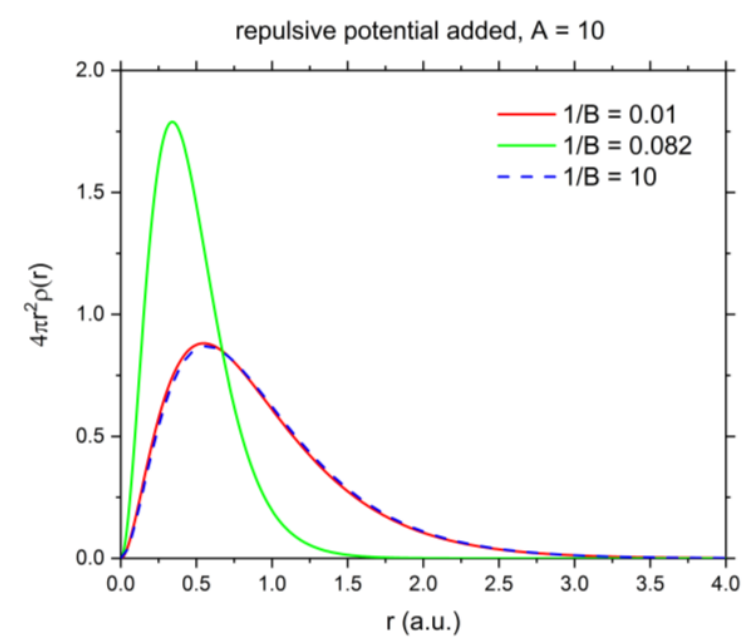

Figure 12. The electron density for the ground state helium with different cavity width $(1 / B)$ of repulsive confinement as a function of radius. The one for $1 / B=10$ (large width limit) is represented in blue dash since it is very similar to the red one for $1 / B=0.01$ (small width limit). The green curve $(1 / B=0.082$, the red valley in Figure 11$)$ shows the most localized behavior.

\subsection{Shannon Entropy for $1 \mathrm{~s} 2 \mathrm{~s}^{1} \mathrm{~S}^{e}$ Excited State of He under Confinement with Repulsive Potential}

We show the energy curve for the $1 \mathrm{~s} 2 \mathrm{~s}{ }^{1} \mathrm{~S}^{\mathrm{e}}$ excited state in Figure 13. Hylleraas-type wave functions with $\omega=13$ ( $N=308$ terms) are employed. At the small cavity limit, the energy is increased by $2 A$, similar to the effect on the ground state. Furthermore, as a consequence of the extra node of the $1 \mathrm{~s} 2 \mathrm{~s}{ }^{1} \mathrm{~S}^{\mathrm{e}}$ excited-state wave function, the wave function interferes with the cavity differently and reflects on Shannon entropy as a "tilt" in Figure 14. The behavior of electron density also justifies such phenomenon. As shown in Figure 15 , at the small $(1 / B=0.004)$ cavity width limit, the electron density is as delocalized as the free helium. The smaller width to the cavity, the more localized it is to the electron density. Eventually, the electron density is the most condensed toward the origin when $1 / B=0.204$. Some selected values of energy and Shannon entropy are listed in Table 5. 


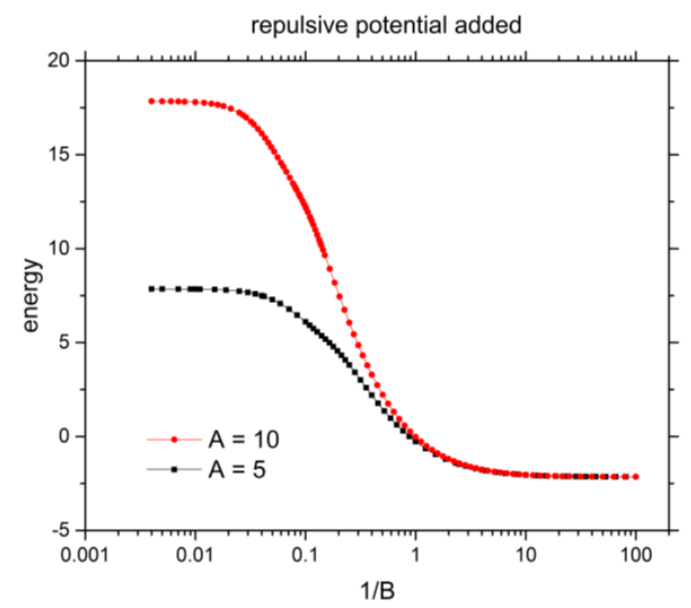

Figure 13. The 1s2s state energy of helium with repulsive finite confinement as a function of the cavity size $1 / B$.

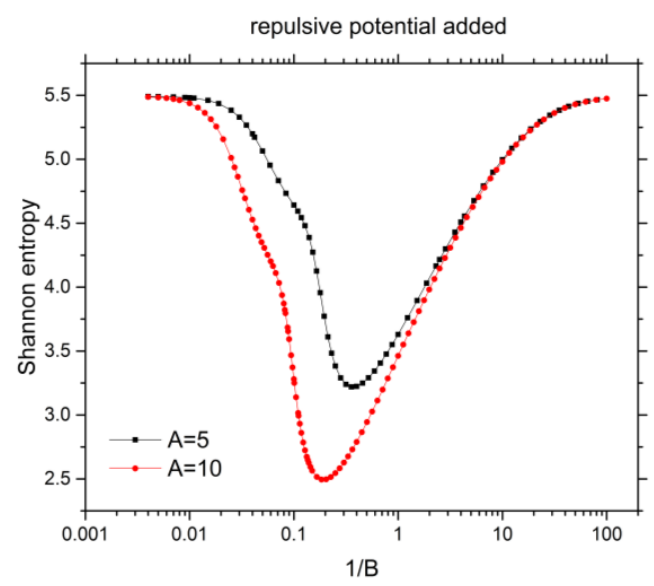

Figure 14. Shannon entropy in position space for the 1s2s state helium with repulsive confinement, shown as a function of the cavity size, $1 / B$. The "tilt" in the left part of the figure is due to the interference with the potential well.

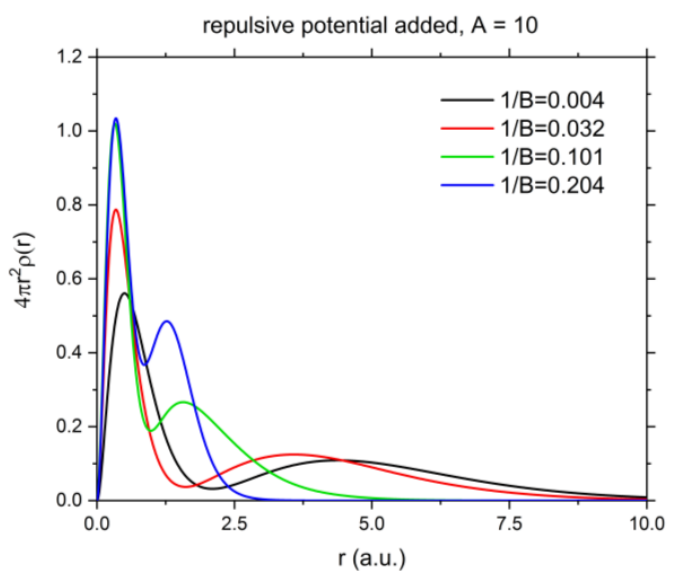

Figure 15. Electron density of $1 \mathrm{~s} 2 \mathrm{~s}^{1} \mathrm{~S}^{\mathrm{e}}$ excited-state helium with attractive confinement of $A=10$ and various $1 / B$ as a function of radius. The black curve is the small cavity limit, where the electrons are more delocalized. The blue curve $(1 / B=0.204)$ corresponds to the valley point $(A=10)$ in Figure 14 , and is the most localized and concentrated. 
Table 5. The 1s2s state energy and Shannon entropy of helium confined with repulsive potentials, with $\omega=13$.

\begin{tabular}{ccccc}
\hline \multirow{2}{*}{$\mathbf{1} \boldsymbol{B}$} & \multicolumn{2}{c}{ Energy } & \multicolumn{2}{c}{ Shannon Entropy } \\
\cline { 2 - 5 } & $\boldsymbol{A}=\mathbf{5}$ & $\boldsymbol{A}=\mathbf{1 0}$ & $\boldsymbol{A}=\mathbf{5}$ & $\boldsymbol{A}=\mathbf{1 0}$ \\
\hline 0.004 & 7.853198946 & 17.849560882 & 5.4911371 & 5.4875642 \\
0.01 & 7.843185873 & 17.797383638 & 5.4813847 & 5.4376764 \\
0.025 & 7.736312535 & 17.238747505 & 5.3837869 & 5.0114612 \\
0.04 & 7.499987880 & 16.12763973 & 5.1996113 & 4.5276297 \\
0.1 & 6.106023017 & 12.21540359 & 4.6418609 & 3.2801230 \\
0.25 & 3.806886884 & 6.058810732 & 3.3829537 & 2.5449126 \\
0.4 & 2.201153744 & 3.294161749 & 3.2239305 & 2.7892007 \\
1 & -0.268402783 & -0.022735449 & 3.6292016 & 3.4623643 \\
2.5 & -1.479882907 & -1.429647473 & 4.2163710 & 4.1451505 \\
4 & -1.780154947 & -1.758998575 & 4.5086622 & 4.4631793 \\
10 & -2.047704730 & -2.044347012 & 4.9971967 & 4.9805825 \\
39.685 & & -2.136636813 & & 5.4009833 \\
43.288 & -2.138141127 & & 5.41429737 & \\
81.113 & -2.143623958 & & 5.46598441 & \\
100 & & -2.144404056 & & 5.4742044 \\
\hline
\end{tabular}

\section{Summary and Conclusions}

By employing highly correlated Hylleraas functions, we have carried out first-time calculations on Shannon information entropy in position space, $S_{r}$, of the ground state and singly excited state for a two-electron helium atom confined with an external finite potential. Both attractive and repulsive confinement potentials in the form of finite oscillatory potentials have been investigated. By unifying the two potentials in this context, a thorough and detailed comparison is made. Furthermore, it is found that for the same set of confinement parameters $(A$ and $1 / B)$, the energy of a specific state under repulsive confinement is larger than that under attractive confinement by $2 A$ while entropies for both cases remain the same. It is concluded that two potentials could provide similar confinement effects if the parameters are the same.

Through systematic examination of convergence behaviors for $S_{r}$ when different expansion lengths in the wave functions are used, we are able to provide benchmark values for Shannon entropy in position space for helium confined by the abovementioned external potentials. Furthermore, for the $1 \mathrm{~s} 2 \mathrm{~s}^{1} \mathrm{~S}^{\mathrm{e}}$ excited state with which wave function processes an additional node as compared to that for the ground state, a "tilt" in the Shannon entropy curve is observed. It seems that Shannon entropy is capable of probing the subtle change of electron localization. Justified by the behavior of the electron density, the localization and delocalization of confined helium have been examined and discussed.

Finally, we should mention that several topics are expected for further research. First, aside from Shannon entropy, other kinds of entropy provide different aspects and understandings for the system. For instance, a relation between natural orbital occupation numbers and Jaynes entropy is reported by March et al. [40]. Second, it is possible to probe the correlation effect by examining the behavior of Shannon entropies under different confinement conditions [41]. Third, Amovilli, Howard and March have systematically investigated the electron density expression for He-like ion sequence with a modified interparticle interaction [42-44]. Therefore, it is possible to examine the behavior of Shannon entropies for these systems. Fourth, in the present work we only calculate the Shannon entropy in position space, $S_{r}$. In order to test the entropic uncertainty principle [45] for an atomic state in three-dimensional space, the Shannon entropy in corresponding momentum space, $S_{p}$, should also be calculated, with

$$
S_{p}=-\int_{0}^{\infty} \gamma(p) \ln \gamma(p) 4 \pi p^{2} d p
$$


where $\gamma(p)$ denotes the momentum density, normalized to unity. Defining $S_{T}$ as the entropy sum, the entropic uncertainty principle is expressed as

$$
S_{T}=S_{r}+S_{p} \geq 3(1+\ln \pi)
$$

The entropy sum can be used to test a stronger version of the Heisenberg's uncertainty principle for an $N$-electron system [46], with

$$
S_{T} \geq 3 N(1+\ln \pi)-2 N \ln N
$$

Saturation of this inequality has been found with Shannon entropy for a harmonic oscillator by Majernik et al. [47]. It remains an interesting and challenging problem to quantify Shannon entropy in momentum space for two-electron states using highly correlated wave functions, and such a calculation is outside the scope of our present investigation. Nevertheless, it is hoped that the findings in our present work would stimulate further investigations on such an intriguing behavior for the highly correlated two-electron system confined by external finite potentials.

Acknowledgments: Jen-Hao Ou would like to thank Chien-Hao Lin and Yen-Chang Lin for helpful discussions and Institute of Atomic and Molecular Sciences, Academia Sinica for granting the internship. We thank the anonymous reviewers for helpful suggestions. Our work was supported by the Ministry of Science and Technology of Taiwan.

Author Contributions: All authors have made contributions leading to the results presented in the present work. All authors have read and approved the final manuscript.

Conflicts of Interest: The authors declare no conflict of interest.

\section{References}

1. Nielsen, M.A.; Chuang, I.L. Quantum Computation and Quantum Information; Cambridge University Press: Cambridge, UK, 2010.

2. Dehesa, J.S.; Koga, T.; Yanez, R.J.; Plastino, A.R.; Esquivel, R.O. Quantum entanglement in helium. J. Phys. B At. Mol. Opt. Phys. 2012, 45, 015504. [CrossRef]

3. Dehesa, J.S.; Koga, T.; Yanez, R.J.; Plastino, A.R.; Esquivel, R.O. Corrigendum: Quantum entanglement in helium. J. Phys. B At. Mol. Opt. Phys. 2012, 45, 239501.

4. Benenti, G.; Siccardi, S.; Strini, G. Entanglement in helium. Eur. Phys. J. D 2013, 67, 1. [CrossRef]

5. Koscik, P. Entanglement in $\mathrm{S}$ states of two-electron quantum dots with Coulomb impurities at the center. Phys. Lett. A 2013, 377, 2393. [CrossRef]

6. Koscik, P.; Okopiska, A. Entanglement Entropies in the Ground States of Helium-Like Atoms. Few-Body Syst. 2014, 55, 1151-1157. [CrossRef]

7. Huang, Z.; Wang, H.; Kais, S. Entanglement and Electron Correlation in Quantum Chemistry Calculations. J. Mod. Opt. 2006, 53, 2543-2558.

8. Hofer, H.T. On the basis set convergence of electron-electron entanglement measures: Helium-like systems. Front. Chem. 2013, 1, 24. [CrossRef] [PubMed]

9. Lopez-Rosa, S.; Esquivel, R.O.; Plastino, A.R.; Dehesa, J.S. Quantum entanglement of helium-like systems with varying-Z: Compact state-of-the-art CI wave functions. J. Phys. B 2015, 48, 175002.

10. Restrepo Cuartas, J.P.; Sanz-Vicario, J.L. Information and entanglement measures applied to the analysis of complexity in doubly excited states of helium. Phys. Rev. A 2015, 91, 052301. [CrossRef]

11. Peng, H.-T.; Ho, Y.K. Statistical Correlations of the N-particle Moshinsky Model. Entropy 2015, 17, $1882-1895$. [CrossRef]

12. Peng, H.T.; Ho, Y.K. Entanglement for excited states of ultracold bosonic atoms in one-dimensional harmonic traps with contact interaction. Mod. Phys. Lett. B 2015, 29, 1550189. [CrossRef]

13. Lin, Y.-C.; Lin, C.-Y.; Ho, Y.K. Spatial entanglement in two-electron atomic systems. Phys. Rev. A 2013, 87, 022316. [CrossRef]

14. Lin, Y.C.; Ho, Y.K. Quantum entanglement for two electrons in the excited states of helium-like systems. Can. J. Phys. 2015, 93, 646-653. [CrossRef] 
15. Lin, C.H.; Lin, Y.C.; Ho, Y.K. Quantification of linear entropy for quantum entanglement in $\mathrm{He}, \mathrm{H}^{-}$and $\mathrm{Ps}^{-}$ ions using highly-correlated Hylleraas functions. Few-Body Syst. 2013, 54, 2147-2153. [CrossRef]

16. Lin, C.H.; Ho, Y.K. Quantification of entanglement entropy in helium by the Schmidt-Slater decomposition method. Few-Body Syst. 2014, 55, 1141-1149. [CrossRef]

17. Lin, C.H.; Ho, Y.K. Calculation of von Neumann entropy for hydrogen and positronium negative ions. Phys. Lett. A 2014, 378, 2861-2865. [CrossRef]

18. Lin, C.H.; Ho, Y.K. Quantification of entanglement entropies for doubly excited states in Helium. Few-Body Syst. 2015, 56, 157. [CrossRef]

19. Lin, C.H.; Ho, Y.K. Quantum Entanglement and Shannon Information Entropy for the Doubly Excited Resonance State in Positronium Negative Ion. Atoms 2015, 3, 422-432. [CrossRef]

20. Lin, Y.C.; Fang, T.K.; Ho, Y.K. Quantum entanglement for helium atom in the Debye plasmas. Phys. Plasmas 2015, 22, 032113.

21. Lin, C.H.; Ho, Y.K. Shannon information entropy in position space for two-electron systems. Chem. Phys. Lett. 2015, 633, 261-264. [CrossRef]

22. Sen, K.D. Characteristic features of Shannon information entropy of confined atoms. J. Chem. Phys. 2005, 123, 074110. [CrossRef] [PubMed]

23. Koscik, P.; Saha, J.K. Entanglement in helium atom confined in an impenetrable cavity. Eur. Phys. J. D 2015, 69, 250. [CrossRef]

24. Koscik, P.; Saha, J.K. Ground-State Entanglement Properties of Helium Atom in a Finite Spherical Cavity. Few-Body Syst. 2015, 56, 645-649. [CrossRef]

25. Sil, A.N.; Canuto, S.; Mukherjee, P.K. Spectroscopy of confined atomic systems: Effect of plasma. Adv. Quantum Chem. 2009, 58, 115.

26. Sen, K.D. (Ed.) Electronic Structure of Quantum Confined Atoms and Molecules; Springer: Cham, Switzerland, 2014.

27. Kimani, P.; Jones, P.; Winkler, P. Correlation studies in weakly confining quantum dot potential. Int. J. Quantum Chem. 2008, 108, 2763. [CrossRef]

28. Chakraborty, S.; Ho, Y.K. Autoionization resonance states of two electron atomic systems with finite spherical confinement. Phys. Rev. A 2011, 84, 032515. [CrossRef]

29. Jiao, L.G.; Ho, Y.K. Bound and Resonant States in Confined Atoms. In Electronic Structure of Quantum Confined Atoms and Molecules; Sen, K.D., Ed.; Springer: Cham, Switzerland, 2014; p. 145.

30. Lin, C.Y.; Ho, Y.K. Photoionization cross sections of hydrogen impurities in spherical quantum dots using the finite-element discrete-variable representation. Phys. Rev. A 2011, 84, 023407. [CrossRef]

31. Lin, C.Y.; Ho, Y.K. Photoionization of endohedral atoms in fullerene cages. Few-Body Syst. 2013, 54, 425-429.

32. Shannon, C.E. A mathematical theory of communication. Bell Syst. Tech. J. 1948, 27, 379-423. [CrossRef]

33. Nagy, A.; Liu, S.B. Local wave-vector, Shannon and Fisher information. Phys. Lett. A 2008, $372,1654$. [CrossRef]

34. Guevara, N.L.; Sagar, R.P.; Esquivel, R.O. Shannon-information entropy sum as a correlation measure in atomic systems. Phys. Rev. A 2003, 67, 012507. [CrossRef]

35. Sagar, R.P.; Laguna, H.G.; Guevara, N.L. Conditional entropies and position-momentum correlations in atomic systems. Mol. Phys. 2009, 107, 2071-2080. [CrossRef]

36. Gadre, S. Information entropy and Thomas-Fermi theory. Phys. Rev. A 1984, 30, 620-621.

37. Gadre, S.; Sears, S.; Chakravorty, S.; Bendale, R. Some novel characteristics of atomic information entropies. Phys. Rev. A 1985, 32, 2602-2606. [CrossRef]

38. Yánez, R.J.; Van Assche, W.; Dehesa, J.S. Position and momentum information entropies of the D-dimensional harmonic oscillator and hydrogen atom. Phys. Rev. A 1994, 50, 3065-3079. [CrossRef] [PubMed]

39. Drake, G.W.F. Atomic, Molecular, and Optical Physics Handbook; AIP Press: New York, NY, USA, 2006; Chapter 11.

40. March, N.; Angilella, G.; Pucci, R. Natural Orbitals in Relation to Quantum Information Theory: From Model Light Atoms through to Emergent Metallic Properties. Int. J. Mod. Phys. B 2013, 27, 1330021.

41. Amovilli, C.; March, N.H. Quantum information: Jaynes and Shannon entropies in a two-electron entangled artificial atom. Phys. Rev. A 2004, 69, 054302. [CrossRef]

42. Amovilli, C.; March, N.H. Density matrices in direct and momentum space for a model of the He-like sequence of atomic ions. Phys. Rev. A 2005, 72, 042504.

43. Howard, I.; March, N.H. Towards a differential equation for the nonrelativistic ground-state electron density of the He-like sequence of atomic ions. Phys. Rev. A 2005, 71, 042501. [CrossRef] 
44. Amovilli, C.; Howard, I.; March, N.H. Analytic inhomogeneous electron liquid and its density for model spin-compensated two-electron atomic ions with Coulomb confinement: An exact nonrelativistic Hamiltonian. Phys. Chem. Liq. 2008, 46, 238-241. [CrossRef]

45. Guevara, N.L.; Sagar, R.P.; Esquivel, R.O. Information uncertainty-type inequalities in atomic systems. J. Chem. Phys. 2003, 119, 7030-7036. [CrossRef]

46. Bialynicki-Birula, I.; Mycielski, J. Uncertainty relations for information entropy in wave mechanics. Commun. Math. Phys. 1975, 44, 12. [CrossRef]

47. Majernik, V.; Opatrný, T. Entropic uncertainty relations for a quantum oscillator. J. Phys. Math. Gen. 1996, 29, 2187-2197. [CrossRef] article distributed under the terms and conditions of the Creative Commons Attribution (CC BY) license (http://creativecommons.org/licenses/by/4.0/). 